\title{
Systematic review and network meta-analysis comparing palbociclib with chemotherapy agents for the treatment of postmenopausal women with HR-positive and HER2-negative advanced/metastatic breast cancer
}

\author{
Florence R. Wilson ${ }^{1} \cdot$ Abhishek Varu ${ }^{1} \cdot$ Debanjali Mitra $^{2} \cdot$ Chris Cameron $^{1} \cdot$ \\ Shrividya Iyer ${ }^{2}$
}

Received: 25 January 2017/ Accepted: 15 July 2017/Published online: 27 July 2017

(c) The Author(s) 2017. This article is an open access publication

\begin{abstract}
Purpose To compare palbociclib + letrozole and palbociclib + fulvestrant with chemotherapy agents in postmenopausal women with hormone receptor-positive $(\mathrm{HR}+) /$ human epidermal growth factor receptor 2-negative (HER2-) advanced/metastatic breast cancer (ABC/MBC) who had no prior systemic treatment for advanced disease (first line) or whose disease progressed after prior endocrine therapy or chemotherapy (second line).

Methods A systematic search identified randomized controlled trials (RCTs) published from January 2000 to January 2016 that compared endocrine-based therapies, chemotherapy agents, and/or chemotherapy agents + biological therapies in the first- and second-line treatment of postmenopausal women with HR+/HER2- ABC/MBC. The main outcome of interest was progression-free survival (PFS)/time to progression (TTP). Bayesian network meta-
\end{abstract}

Electronic supplementary material The online version of this article (doi:10.1007/s10549-017-4404-4) contains supplementary material, which is available to authorized users.

\author{
Chris Cameron \\ ccameron@cornerstone-research.com \\ Florence R. Wilson \\ fwilson@cornerstone-research.com \\ Abhishek Varu \\ avaru@cornerstone-research.com \\ Debanjali Mitra \\ debanjali.mitra@pfizer.com \\ Shrividya Iyer \\ shrividya.iyer@pfizer.com \\ 1 Cornerstone Research Group Inc., Suite 204, 3228 South \\ Service Road, Burlington, ON L7N 3H8, Canada \\ 2 Pfizer, Inc., New York, NY, USA
}

analyses (NMAs) and pairwise meta-analyses were conducted. Heterogeneity and inconsistency were assessed. Results Sixty RCTs met eligibility criteria and were stratified by line of therapy. In the first line, palbociclib + letrozole showed statistically significant improvements in PFS/TTP versus capecitabine [intermittent: HR 0.28 (95\% CrI 0.11-0.72)] and mitoxantrone [HR 0.28 (0.13-0.61)], and trended toward improvements versus paclitaxel [HR 0.59 (0.19-1.96)], docetaxel [HR 0.51 (0.14-2.03)] and other monotherapy or combination agents (HRs ranging from 0.24 to 0.99 ). In the second line, palbociclib + fulvestrant showed statistically significant improvements in PFS/TTP versus capecitabine [intermittent: HR 0.28 (0.13-0.65)], mitoxantrone [HR 0.26 $(0.12-0.53)]$, and pegylated liposomal doxorubicin [HR $0.19(0.07-0.50)]$, and trended toward improvements versus paclitaxel [HR 0.48 (0.16-1.44)], docetaxel [HR 0.71 (0.24-2.13)] and other monotherapy or combination agents (HRs ranging from 0.23-0.89). NMA findings aligned with direct evidence and were robust to sensitivity analyses.

Conclusions Palbociclib + letrozole and palbociclib + fulvestrant demonstrate trends in incremental efficacy compared with chemotherapy agents for the first- and second-line treatment of HR +/HER2- ABC/MBC.

Keywords Advanced/metastatic breast cancer . Chemotherapy · Network meta-analysis · Palbociclib . Progression-free survival - Time to progression

$\begin{array}{ll}\text { Abbreviations } \\ \text { ABC } & \text { Advanced breast cancer } \\ \text { HER2 }- & \begin{array}{l}\text { Human epidermal growth factor receptor type 2 } \\ \text { negative }\end{array} \\ \text { HR+ } & \begin{array}{l}\text { Hormone receptor positive } \\ \text { MBC }\end{array} \quad \text { Metastatic breast cancer }\end{array}$


NMA Network meta-analysis

PFS Progression-free survival

RCT Randomized control trial

TTP Time to progression

\section{Introduction}

Postmenopausal women with hormone receptor-positive $(\mathrm{HR}+)$, human epidermal growth factor receptor type 2-negative (HER2-) tumors represent the majority of patients with advanced/metastatic breast cancer (ABC/ $\mathrm{MBC}$ ) [1-3]. Despite the sometimes indolent course of the disease, HR+/HER2 - ABC/MBC remains incurable [1-3]. Guidelines suggest that endocrine therapy should be offered as standard first-line treatment in patients who do not have visceral crises [1-3]. After receiving first-line endocrine therapy, many patients experience disease progression due to endocrine resistance and are offered chemotherapy as second-line therapy [2]. Various monotherapy and combination chemotherapy regimens are available, providing treatment options for patients with endocrine resistance [4].

Palbociclib (IBRANCE ${ }^{\circledR}$; Pfizer Inc, New York, NY, USA) is a new oral cyclin-dependent kinase 4/6 (CDK4/6) inhibitor approved by the United States (US) Food and Drug Administration (FDA) for $\mathrm{HR}+/ \mathrm{HER} 2-\mathrm{ABC} / \mathrm{MBC}$ in combination with letrozole as initial endocrine-based therapy [5], or in combination with fulvestrant for patients whose disease had progressed following prior endocrine therapy [6]. The efficacy and safety of palbociclib combination therapies have been demonstrated in phase 3 clinical studies $[5,6]$; however, a comparison of progression-free survival (PFS) has not been made between palbociclib and chemotherapy agents. Here, we report the results of a systematic literature review (SLR) and network meta-analysis (NMA) that evaluates the efficacy of palbociclib + letrozole and palbociclib + fulvestrant versus chemotherapy agents in postmenopausal women with $\mathrm{HR}+/ \mathrm{HER} 2-\mathrm{ABC} /$ $\mathrm{MBC}$ who had no prior systemic treatment for advanced disease (first line) or whose disease had progressed after prior endocrine therapy or chemotherapy (second line).

\section{Methods}

\section{Systematic literature review}

An SLR was conducted to identify randomized controlled trials (RCTs) published from January 2000 to January 2016. All references used in two previous NMAs by Generali et al. [7] and Chirila et al. [8] formed the starting point for the current systematic review. These represent the most recent
NMAs conducted for chemotherapy agents and for endocrine therapies. The NMA by Generali et al. [7] compared everolimus + exemestane with various chemotherapy agents, and the literature search spanned from 2000 to May 2014. The NMA by Chirila et al. [8] compared palbociclib with other endocrine-based therapies, and the literature search was conducted in January 2015 with no date restrictions. An updated literature search was performed by searching MEDLINE, EMBASE, Cochrane CENTRAL, and PubMed from May 2014 (search date of Generali) to January 2016 to identify RCTs that were published since the aforementioned two reviews. A predefined search strategy (Online Appendix A) was used, based on the previous searches by Generali et al. [7] and Chirila et al. [8]. The search was designed to identify all RCTs of chemotherapy agents, chemotherapy agents + biological therapies, and endocrine therapies used to treat postmenopausal women with $\mathrm{HR}+$ / HER2 - ABC/MBC who had not received any prior systemic anticancer treatment for advanced disease (first line) or whose disease had progressed after prior endocrine therapy or chemotherapy (second line). However, the current analysis focuses only on chemotherapy agents.

Predefined eligibility criteria were used to screen all identified studies (Online Appendix B; additional details are available upon request). Phase 2 and phase 3 RCTs and conference abstracts were included. Treatments of interest included chemotherapy agents, chemotherapy agents + biological therapies, and endocrine-based therapies. Endocrine-based therapies were included in all analyses but have not been reported here, given that the focus of this analysis is on chemotherapy agents. Outcomes of interest were PFS, time to progression (TTP), and overall survival (OS), reported as hazard ratios (HRs) with $95 \%$ confidence intervals (CIs). PFS and TTP were considered as equivalent outcomes since the definitions aligned well across studies and any heterogeneity was considered non-substantial. As the outcome of disease progression is a negative event for patients, HRs $<1$ corresponded to beneficial treatment effects of the first treatment compared with the second treatment. The analysis of OS has been excluded here due to lack of availability of final OS data from the palbociclib clinical trials.

Two reviewers independently reviewed citation titles and abstracts identified in the updated literature search to assess study eligibility. Citations considered to describe potentially eligible articles were independently reviewed in full-text form. A PRISMA flow diagram documenting the process of study selection was prepared.

\section{Network meta-analysis}

Network meta-analysis is a widely used approach to derive estimates of effect among treatments that may not have 
been compared directly in clinical trials. Bayesian NMAs and pairwise meta-analyses were conducted to pool RCT results using well-established methods outlined by the National Institute for Health and Care Excellence (NICE) $[9,10]$. Two separate evidence networks were generated to stratify studies by first and second lines of therapy. Based on the line of therapy definitions used in the palbociclib clinical trials [5, 6], first line of therapy was defined as having neither previous systemic endocrine therapy nor chemotherapy for $\mathrm{ABC} / \mathrm{MBC}$, and second line of therapy was defined as having previous systemic endocrine therapy or chemotherapy for $\mathrm{ABC} / \mathrm{MBC}$.

For each pairwise comparison, HRs with $95 \%$ credible intervals (CrIs) were used as a measure of the association between the treatment and its efficacy. Estimates with $95 \%$ CrIs that excluded the null value of 1 were considered to reflect statistically significant differences between interventions. Additional measures of effect were also generated, including Surface Under the Cumulative RAnking curve (SUCRA) values (expressed as percentages, which show the relative probability of an intervention being among the best options), probability best, and mean rank [11]. For interpretation, SUCRA values and probability best range between 0 and 1 , with values closer to 1 being preferred [11].

Fixed-effects models were performed as primary analyses, given that the networks are largely composed of single-study connections. Random-effects models were performed as secondary analyses, using both informative and vague priors on the variance. Informative priors were based on an estimate of between-study variance using data from previous Cochrane systematic reviews [12]. For vague priors, we assumed a uniform distribution [i.e., Uniform $(0,5)]$ for between-study variance, as recommended by the NICE [9]. To assess whether the models had adequate fit to the data, the posterior residual deviance from each NMA was compared to the corresponding number of unconstrained data points; approximately equal values represented an adequate fit.

Network meta-analyses were performed using WinBUGS (version 1.4.3) and R (version 3.2.2) and were based on burn-in samples of at least 40,000 iterations and subsequent sampling iterations of at least 50,000 iterations (WinBUGS code is available upon request). Trace plots and Gelman-Rubin plots were reviewed to assess model convergence.

\section{Assessment of heterogeneity and inconsistency}

In accordance with the exchangeability assumption of NMAs [13], study and patient characteristics were assessed to ensure similarity and to investigate the potential impact of heterogeneity on effect estimates. Factors considered included mean/median age, HR status, HER2 status, menopausal status, prior therapies, crossover after disease progression, blinding, drug dosing, and endpoint definitions. Heterogeneity was assessed by summarizing relevant information using tables and by conducting sensitivity analyses where possible. The presence of several singlestudy connections between interventions in the evidence networks precluded us from performing meta-regression analyses or sub-group/sensitivity analyses related to certain characteristics of interest [14]. Sensitivity analyses were conducted to include both the palbociclib phase 2 and 3 studies [5, 15], and to adjust for heterogeneity in median PFS/TTP values.

The NMA results were qualitatively compared with pairwise estimates generated from traditional frequentist meta-analyses of direct evidence. Inconsistency in the networks was assessed by comparing deviance and deviance information criterion (DIC) statistics in fitted consistency and inconsistency models [16]. The posterior mean deviance of the individual data points in the inconsistency model was plotted against the corresponding posterior mean deviance in the consistency model to identify any loops where inconsistency was present (available upon request).

\section{Results}

\section{Study selection}

The NMA by Generali et al. [7] included 44 RCTs, which were not stratified by line of therapy. The NMA by Chirila et al. [8] included 27 RCTs, stratified by line of therapy. Of these, 53 RCTs met the eligibility criteria described above. In addition, two recently published studies that provided updated PFS results for palbociclib trials in first line [5] and second line [6] were included. Among the 2600 study records that were identified in the updated literature search, seven RCTs met the eligibility criteria and were included in the NMA.

In total, 60 RCTs (from the three SLRs) met the eligibility criteria; however, only 57 RCTs were included in the PFS/TTP NMA that is presented here (Fig. 1). In order for the evidence networks to be fully connected, some connections had to be forced based on line of therapy and patient characteristics. The three connections forced based on patient characteristics were chemotherapy trials and were due to less than $50 \%$ of patients being HR+ [17-19].

\section{Study and patient characteristics}

The 57 RCTs included in analyses were published between 1992 and 2016, with trials conducted on all 


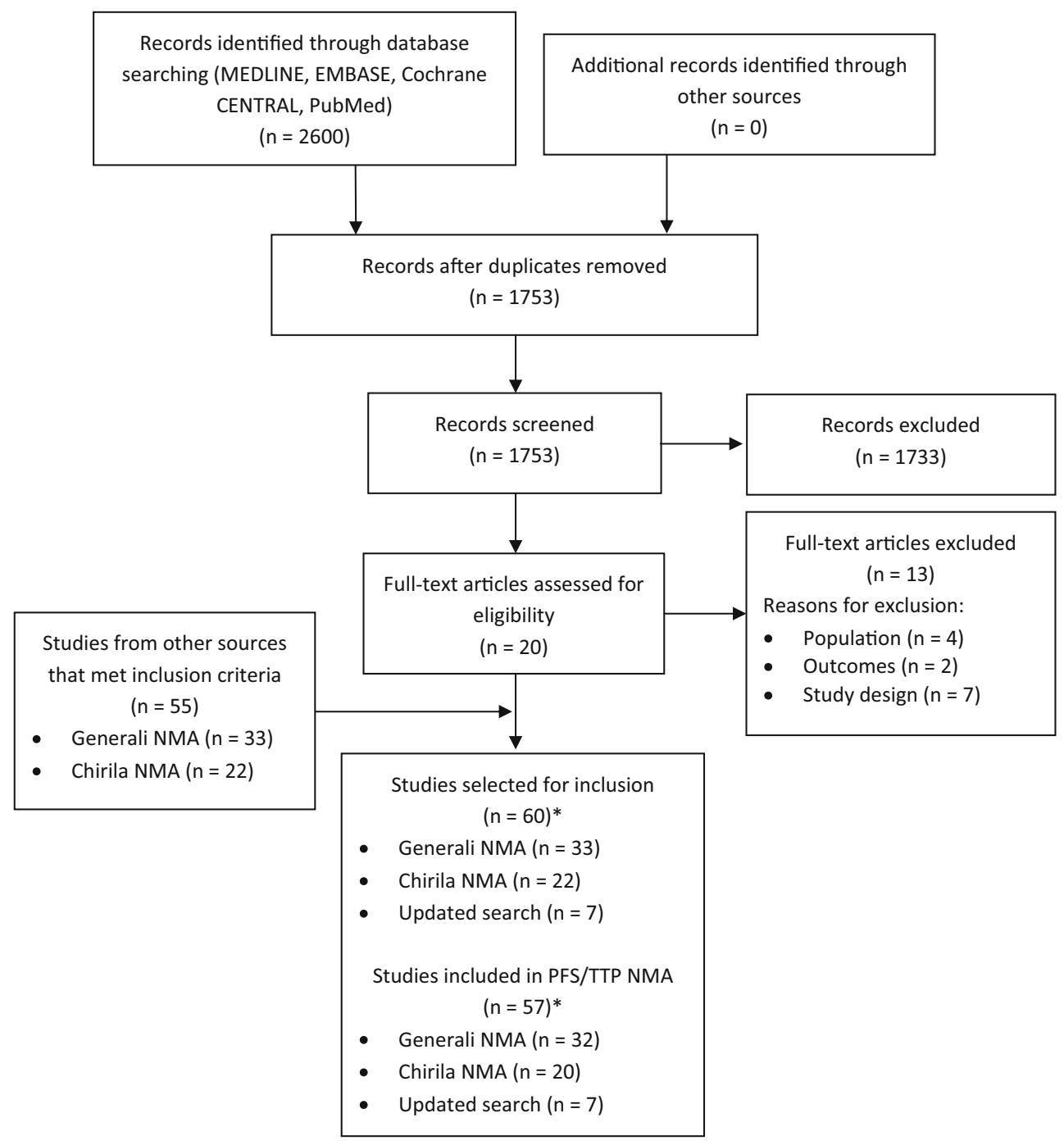

Fig. 1 PRISMA flow diagram NMA network meta-analysis, PRISMA preferred reporting items for systematic reviews and meta-analyses. *Two studies overlapped between the Generali et al. NMA and the Chirila et al. NMA

continents. Mean age across the trials ranged from 51 to 70 years, and median follow-up ranged from 6 to 61.2 months (Online Appendix C). The percentage of $\mathrm{HR}+$ patients was reported in 56 of the 57 trials and ranged from about 15 to $100 \%$, and the proportion of patients receiving prior metastatic endocrine therapy or chemotherapy ranged from 0 to $100 \%$. Based on this high level of heterogeneity, trials were stratified by line of therapy based on prior neoadjuvant/adjuvant and advanced/metastatic therapy received by patients (details available upon request). Assessment of other study and patient characteristics revealed that many sensitivity analyses were not feasible due to insufficient information or disconnected evidence networks. Overall, the studies included in the NMA had a low risk of bias (Online Appendix D). A summary of the median PFS/TTP values and HRs used in analyses is available upon request.

\section{First-line therapy progression-free survival/time to progression}

The evidence network for the first-line PFS/TTP NMA is shown in Fig. 2. Each intervention is represented by a node and randomized comparisons are shown as links between the nodes. Overall, 22 studies were included that enrolled a total of 8152 patients with available outcomes data. Data from head-to-head trials were available for 28 pairwise comparisons in the network, with single studies informing all of these comparisons. This analysis includes data from the PALOMA-2 trial which compares palbociclib + letrozole with letrozole [5].

In the fixed-effects model, palbociclib + letrozole showed statistically significant improvements in PFS/TTP relative to capecitabine [intermittent: HR 0.28 (95\% $\mathrm{CrI}$ $0.11-0.72)]$ and mitoxantrone [HR $0.28(0.13-0.61)]$, and 


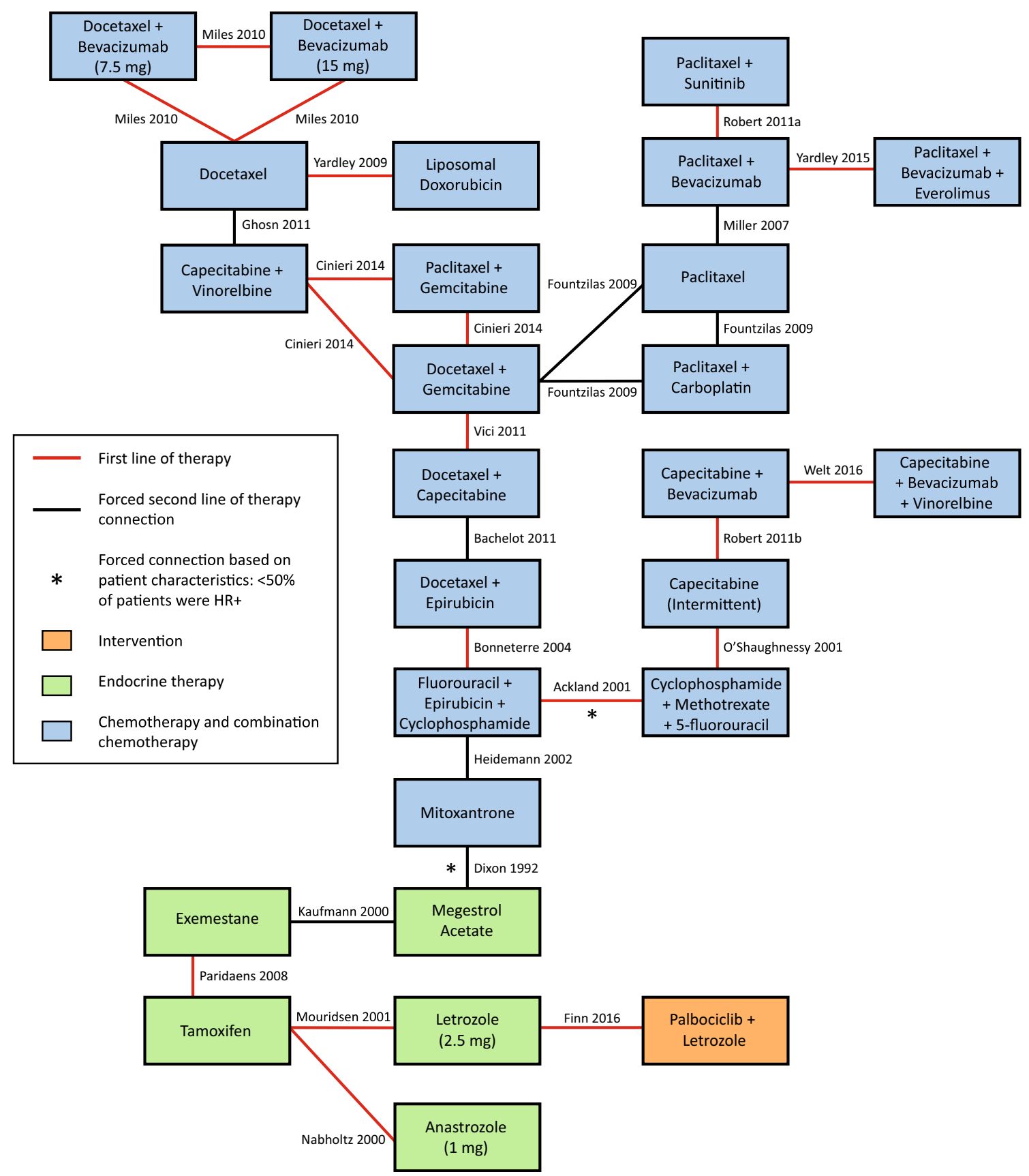

Fig. 2 Evidence network for first-line PFS/TTP $H R+$ hormone receptor positive, $P F S$ progression-free survival, $T T P$ time to progression

trended toward improvements (not statistically significant) versus paclitaxel [HR $0.59(0.19-1.96)$ ], docetaxel (HR $0.51(0.14-2.03)]$, and other monotherapy or combination chemotherapy agents (HRs ranging from 0.24 to 0.99 ; Table 1). Palbociclib + letrozole ranked more favorably than all chemotherapy comparators for PFS/TTP in terms of SUCRA, probability best, and mean rank. Palbociclib + letrozole was associated with the highest SUCRA value among all treatments $(96.00 \%)$, the highest probability of being the best treatment $(41.70 \%)$, and a treatment ranking closest to 1 . Model fit statistics from the fixedeffects model were favorable; a total residual deviance value close to the number of unconstrained data points was obtained (i.e., 25.08 vs. 25 ).

In the random-effects models using both informative and vague priors on the variance, palbociclib + letrozole trended toward improvements (not statistically significant) versus all chemotherapy comparators. Model fit was favorable and relatively constant across both analyses (Table 1). 
Table 1 First-line therapy NMA results for PFS/TTP: palbociclib + letrozole versus comparators

\begin{tabular}{|c|c|c|c|}
\hline Comparisons & $\begin{array}{l}\text { HR }(95 \% \text { CrI }) \\
\text { Fixed-effects model }\end{array}$ & $\begin{array}{l}\text { HR }(95 \% \mathrm{CrI}) \\
\text { Random-effects model: } \\
\text { vague priors }\end{array}$ & $\begin{array}{l}\text { HR }(95 \% \mathrm{CrI}) \\
\text { Random-effects model: } \\
\text { informative priors }\end{array}$ \\
\hline Palbociclib + letrozole & 1 & 1 & 1 \\
\hline \multicolumn{4}{|l|}{ Single chemotherapy agents } \\
\hline Paclitaxel & $0.59(0.19-1.96)$ & $0.59(0.07-4.83)$ & $0.63(0.07-5.48)$ \\
\hline Docetaxel & $0.51(0.14-2.03)$ & $0.50(0.06-3.92)$ & $0.55(0.07-4.24)$ \\
\hline Capecitabine (intermittent) & $0.28(0.11-0.72)$ & $0.27(0.03-2.12)$ & $0.29(0.03-2.45)$ \\
\hline Mitoxantrone & $0.28(0.13-0.61)$ & $0.27(0.03-2.23)$ & $0.28(0.03-2.28)$ \\
\hline \multicolumn{4}{|l|}{ Combination chemotherapy agents } \\
\hline Paclitaxel + bevacizumab + everolimus & $0.99(0.29-3.81)$ & $0.98(0.12-8.43)$ & $0.93(0.12-7.17)$ \\
\hline Paclitaxel + bevacizumab & $0.98(0.31-3.38)$ & $0.96(0.13-7.29)$ & $0.94(0.10-8.65)$ \\
\hline Docetaxel + bevacizumab $15 \mathrm{mg}$ & $0.65(0.18-2.69)$ & $0.65(0.09-4.70)$ & $0.72(0.09-6.11)$ \\
\hline Docetaxel + bevacizumab $7.5 \mathrm{mg}$ & $0.59(0.16-2.40)$ & $0.58(0.08-4.19)$ & $0.64(0.08-5.36)$ \\
\hline Paclitaxel + sunitinib & $0.60(0.18-2.14)$ & $0.60(0.07-4.93)$ & $0.66(0.09-5.00)$ \\
\hline Docetaxel + gemcitabine & $0.59(0.20-1.91)$ & $0.59(0.08-4.32)$ & $0.64(0.08-5.20)$ \\
\hline Liposomal doxorubicin & $0.54(0.14-2.29)$ & $0.53(0.07-3.79)$ & $0.59(0.07-4.99)$ \\
\hline Paclitaxel + gemcitabine & $0.51(0.15-1.87)$ & $0.49(0.06-4.21)$ & $0.56(0.08-4.06)$ \\
\hline Paclitaxel + carboplatin & $0.53(0.17-1.83)$ & $0.51(0.06-4.35)$ & $0.57(0.06-5.16)$ \\
\hline Docetaxel + capecitabine & $0.51(0.19-1.49)$ & $0.50(0.06-4.19)$ & $0.54(0.08-3.90)$ \\
\hline Capecitabine + vinorelbine & $0.50(0.16-1.72)$ & $0.49(0.06-4.18)$ & $0.55(0.06-4.98)$ \\
\hline Capecitabine + bevacizumab + vinorelbine & $0.48(0.19-1.27)$ & $0.46(0.06-3.76)$ & $0.50(0.06-4.02)$ \\
\hline Docetaxel + epirubicin & $0.47(0.20-1.19)$ & $0.46(0.06-3.32)$ & $0.49(0.06-4.15)$ \\
\hline Capecitabine + bevacizumab & $0.40(0.16-1.06)$ & $0.39(0.05-2.82)$ & $0.41(0.06-2.99)$ \\
\hline Fluorouracil + epirubicin + cyclophosphamide & $0.33(0.15-0.77)$ & $0.32(0.04-2.47)$ & $0.34(0.03-3.34)$ \\
\hline Cyclophosphamide + methotrexate +5 -fluorouracil & $0.24(0.11-0.57)$ & $0.24(0.03-2.11)$ & $0.25(0.03-1.85)$ \\
\hline \multirow[t]{3}{*}{ Model fit statistics } & $\begin{array}{l}\text { Residual deviance } \\
=25.08 \text { vs. } 25\end{array}$ & $\begin{array}{l}\text { Residual deviance } \\
=25.71 \text { vs. } 25\end{array}$ & $\begin{array}{l}\text { Residual deviance } \\
=25.69 \text { vs. } 25\end{array}$ \\
\hline & \multirow[t]{2}{*}{$\mathrm{DIC}=-0.02$} & $\mathrm{DIC}=0.11$ & $\mathrm{DIC}=-0.05$ \\
\hline & & $\begin{array}{l}\text { Heterogeneity (SD) } \\
=0.67(0.01-4.53)\end{array}$ & $\begin{array}{l}\text { Heterogeneity (SD) } \\
=0.73(0.02-3.64)\end{array}$ \\
\hline
\end{tabular}

Analyses use data from PALOMA-2 [5]. Statistically significant differences are shown in bold. Endocrine therapies have been excluded from this table, given that the focus is on chemotherapy agents. For vague priors in the random-effects model, a uniform distribution for between-study variance was assumed, as recommended by the National Institute for Health and Care Excellence [9]. Informative priors were based on an estimate of between-study variance using data from previous Cochrane systematic reviews [12]

$C r l$ credible interval, $D I C$ deviance information criterion, $H R$ hazard ratio, $S D$ standard deviation

\section{Second-line therapy progression-free survival/time to progression}

Figure 3 presents the evidence network for the second-line PFS/TTP NMA. Overall, 44 studies were included that enrolled a total of 14,708 patients with available outcomes data. Data from head-to-head trials were available for 45 of the pairwise comparisons in the network, with single studies informing 35 of these comparisons. This analysis includes data from the PALOMA-3 trial which compares palbociclib + fulvestrant with fulvestrant $500 \mathrm{mg}$ [6].

In the fixed-effects model, palbociclib + fulvestrant showed statistically significant improvements in PFS/TTP relative to capecitabine [intermittent: HR 0.28 (95\% $\mathrm{CrI}$ 0.13-0.65); continuous: HR 0.24 (0.11-0.56)], mitoxantrone [HR $0.26(0.12-0.53)$ ], and pegylated liposomal doxorubicin [HR $0.19(0.07-0.50)]$, and trended toward improvements (not statistically significant) versus paclitaxel [HR 0.48 (0.16-1.44)], docetaxel [HR 0.71 (0.24-2.13)], and other monotherapy or combination chemotherapy agents (HRs ranging from 0.23 to 0.89 ; Table 2). Palbociclib + fulvestrant ranked more favorably than all chemotherapy comparators for PFS/TTP in terms of SUCRA, probability best, and mean rank. Palbociclib + fulvestrant was associated with the highest SUCRA value among all treatments $(97.20 \%)$, and an $18.90 \%$ probability of being the best treatment. 


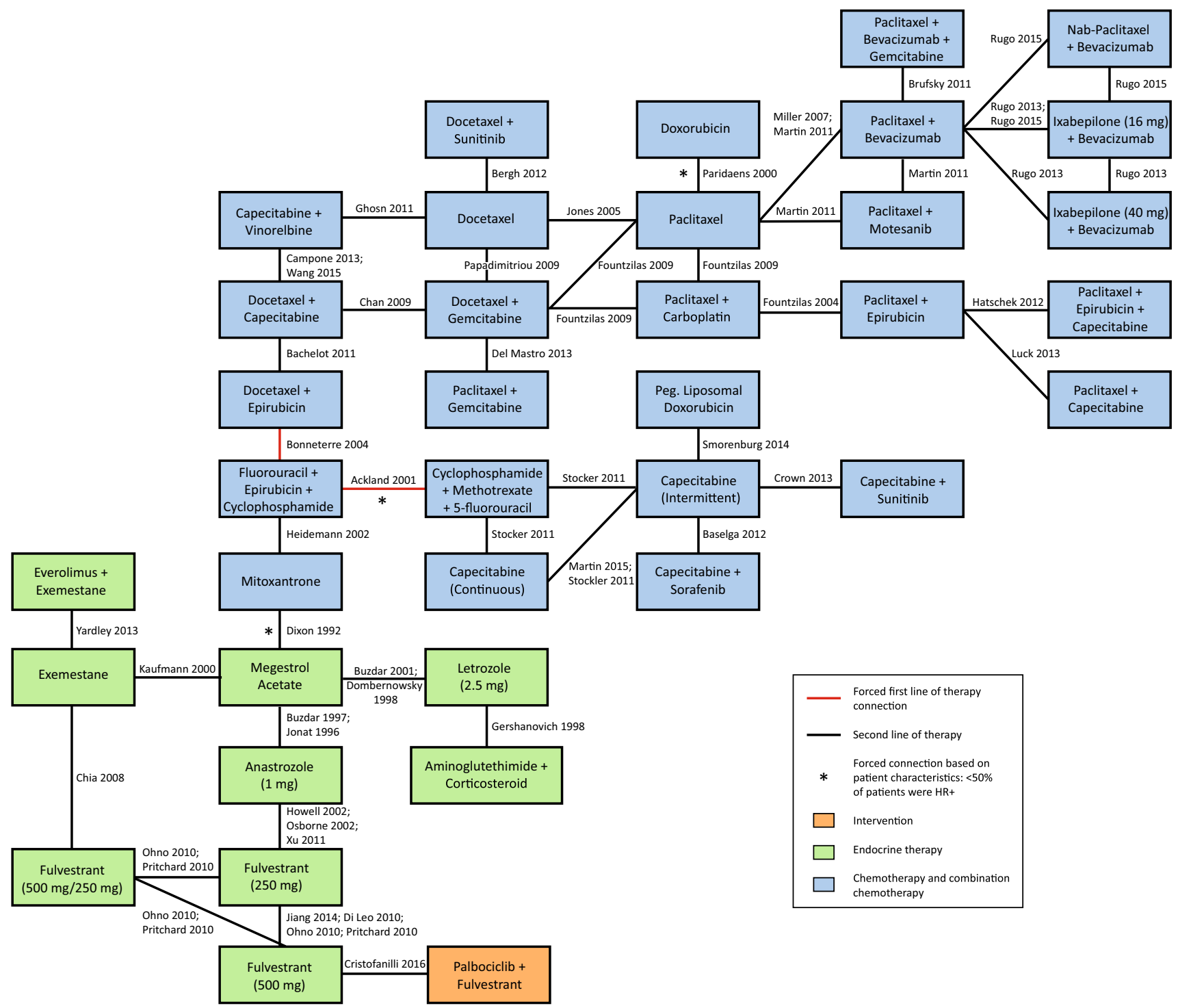

Fig. 3 Evidence network for second-line PFS/TTP $H R+$ hormone receptor positive, $P F S$ progression-free survival, TTP time to progression

Model fit statistics from the fixed-effects model indicated a poor fit; a total residual deviance value greater than the number of unconstrained data points was obtained (i.e., 58.12 vs. 51). This high residual deviance was largely driven by one study [20], which was removed in a sensitivity analysis and model fit improved (Online Appendix E).

In the second-line random-effects model using vague priors, palbociclib + fulvestrant showed statistically significant improvements versus capecitabine [intermittent: HR 0.29 (95\% CrIR 0.10-0.81); continuous: HR 0.25 (0.09-0.70)], mitoxantrone [HR $0.26(0.11-0.60)]$, and pegylated liposomal doxorubicin [HR $0.2(0.06-0.64)$ ] and trended toward improvements versus paclitaxel [HR $0.49(0.14-1.74)]$, docetaxel [HR $0.69(0.2-2.57)]$, and other monotherapy or combination chemotherapy agents (HRs ranging from 0.23 to 0.89 ). Similar results and statistical significance were obtained from the randomeffects model using informative priors. Model fit statistics were favorable from both random-effects models (Table 2).

\section{Sensitivity analyses}

Sensitivity analyses were conducted to include both the palbociclib phase 2 and 3 studies [5, 15], and to adjust for heterogeneity in median PFS/TTP values (Online Appendix E). For each sensitivity analysis in the first line of therapy, palbociclib + letrozole was associated with improved PFS/TTP relative to all other treatments. After 
Table 2 Second-line therapy NMA results for PFS/TTP: palbociclib + fulvestrant versus comparators

\begin{tabular}{|c|c|c|c|}
\hline Comparisons & $\begin{array}{l}\text { HR }(95 \% \mathrm{CrI}) \\
\text { Fixed-effects model }\end{array}$ & $\begin{array}{l}\text { HR }(95 \% \text { CrI }) \\
\text { Random-effects model: } \\
\text { vague priors }\end{array}$ & $\begin{array}{l}\text { HR }(95 \% \text { CrI }) \\
\text { Random-effects model: } \\
\text { informative priors }\end{array}$ \\
\hline Palbociclib + Fulvestrant & 1 & 1 & 1 \\
\hline \multicolumn{4}{|l|}{ Single chemotherapy agents } \\
\hline Doxorubicin & $0.80(0.27-2.44)$ & $0.81(0.22-3.02)$ & $0.82(0.19-3.40)$ \\
\hline Docetaxel & $0.71(0.24-2.13)$ & $0.69(0.20-2.57)$ & $0.70(0.17-2.76)$ \\
\hline Paclitaxel & $0.48(0.16-1.44)$ & $0.49(0.14-1.74)$ & $0.49(0.12-1.98)$ \\
\hline Capecitabine (intermittent) & $0.28(0.13-0.65)$ & $0.29(0.10-0.81)$ & $0.29(0.10-0.89)$ \\
\hline Mitoxantrone & $0.26(0.12-0.53)$ & $0.26(0.11-0.60)$ & $0.26(0.11-0.65)$ \\
\hline Capecitabine (continuous) & $0.24(0.11-0.56)$ & $0.25(0.09-0.70)$ & $0.25(0.09-0.78)$ \\
\hline Pegylated liposomal doxorubicin & $0.19(0.07-0.50)$ & $0.20(0.06-0.64)$ & $0.19(0.06-0.67)$ \\
\hline \multicolumn{4}{|l|}{ Combination chemotherapy agents } \\
\hline Paclitaxel + bevacizumab + gemcitabine & $0.89(0.28-2.82)$ & $0.89(0.23-3.49)$ & $0.91(0.20-3.95)$ \\
\hline Docetaxel + sunitinib & $0.77(0.26-2.36)$ & $0.75(0.21-2.81)$ & $0.76(0.18-3.12)$ \\
\hline Paclitaxel + bevacizumab & $0.72(0.24-2.20)$ & $0.73(0.20-2.68)$ & $0.74(0.17-3.11)$ \\
\hline Paclitaxel + gemcitabine & $0.64(0.22-1.94)$ & $0.67(0.19-2.45)$ & $0.67(0.16-2.77)$ \\
\hline Ixabepilone $40 \mathrm{mg}+$ bevacizumab & $0.61(0.18-2.05)$ & $0.62(0.15-2.53)$ & $0.62(0.13-2.84)$ \\
\hline Nab-paclitaxel + bevacizumab & $0.60(0.20-1.87)$ & $0.60(0.16-2.27)$ & $0.61(0.13-2.61)$ \\
\hline Docetaxel + gemcitabine & $0.55(0.19-1.61)$ & $0.57(0.17-1.99)$ & $0.58(0.15-2.29)$ \\
\hline Paclitaxel + motesanib & $0.51(0.16-1.61)$ & $0.53(0.14-2.13)$ & $0.53(0.12-2.33)$ \\
\hline Docetaxel + capecitabine & $0.49(0.17-1.38)$ & $0.51(0.16-1.70)$ & $0.51(0.14-1.96)$ \\
\hline Docetaxel + epirubicin & $0.45(0.19-1.06)$ & $0.46(0.17-1.28)$ & $0.47(0.16-1.44)$ \\
\hline Paclitaxel + carboplatin & $0.44(0.14-1.38)$ & $0.46(0.13-1.67)$ & $0.46(0.11-1.94)$ \\
\hline Ixabepilone $16 \mathrm{mg}+$ bevacizumab & $0.45(0.15-1.39)$ & $0.45(0.12-1.71)$ & $0.46(0.10-1.93)$ \\
\hline Capecitabine + sorafenib & $0.44(0.17-1.14)$ & $0.45(0.14-1.49)$ & $0.44(0.14-1.54)$ \\
\hline Capecitabine + vinorelbine & $0.44(0.15-1.28)$ & $0.47(0.14-1.62)$ & $0.47(0.13-1.85)$ \\
\hline Paclitaxel + epirubicin & $0.35(0.11-1.16)$ & $0.36(0.09-1.44)$ & $0.31(0.07-1.37)$ \\
\hline Fluorouracil + epirubicin + cyclophosphamide & $0.31(0.14-0.67)$ & $0.32(0.13-0.78)$ & $0.31(0.12-0.87)$ \\
\hline Paclitaxel + capecitabine & $0.3(0.09-0.98)$ & $0.31(0.08-1.23)$ & $0.31(0.07-1.44)$ \\
\hline Paclitaxel + epirubicin + capecitabine & $0.29(0.09-0.95)$ & $0.31(0.08-1.16)$ & $0.37(0.08-1.70)$ \\
\hline Capecitabine + sunitinib & $0.23(0.1-0.56)$ & $0.24(0.08-0.71)$ & $0.23(0.08-0.78)$ \\
\hline $\begin{array}{l}\text { Cyclophosphamide }+ \text { methotrexate } \\
+5 \text {-fluorouracil }\end{array}$ & $0.23(0.1-0.51)$ & $0.23(0.09-0.61)$ & $0.23(0.09-0.68)$ \\
\hline \multirow[t]{3}{*}{ Model fit statistics } & $\begin{array}{l}\text { Residual deviance } \\
=58.12 \text { vs. } 51\end{array}$ & $\begin{array}{l}\text { Residual deviance } \\
=52.50 \text { vs. } 51\end{array}$ & $\begin{array}{l}\text { Residual deviance } \\
=52.11 \text { vs. } 51\end{array}$ \\
\hline & \multirow[t]{2}{*}{$\mathrm{DIC}=-4.11$} & $\mathrm{DIC}=-4.36$ & $\mathrm{DIC}=-4.63$ \\
\hline & & $\begin{array}{l}\text { Heterogeneity (SD) } \\
=0.11(0.01-0.26)\end{array}$ & $\begin{array}{l}\text { Heterogeneity (SD) } \\
=0.11(0.01-0.26)\end{array}$ \\
\hline
\end{tabular}

Analyses use data from PALOMA-3 [6]. Statistically significant differences are shown in bold. Endocrine therapies have been excluded from this table, given that the focus is on chemotherapy agents. For vague priors in the random-effects model, a uniform distribution for between-study variance was assumed, as recommended by the National Institute for Health and Care Excellence [9]. Informative priors were based on an estimate of between-study variance using data from previous Cochrane systematic reviews [12]

$C r I$ credible interval, DIC deviance information criterion, $H R$ hazard ratio, $S D$ standard deviation

adjusting for heterogeneity in median PFS/TTP values in the second-line analysis, palbociclib + fulvestrant was associated with improved PFS/TTP relative to all chemotherapy comparators. Model fit was favorable across all sensitivity analyses.

\section{Discussion}

An SLR and NMAs were conducted to indirectly compare palbociclib + letrozole and palbociclib + fulvestrant with chemotherapy agents used in the first- and second-line 
treatment of postmenopausal women with HR +/HER2$\mathrm{ABC} / \mathrm{MBC}$.

The first-line NMA results suggest that palbociclib + letrozole is associated with improved PFS/TTP relative to all other treatments. In the fixed-effects model, statistically significant improvements in PFS/TTP were observed in favor of palbociclib + letrozole relative to capecitabine (intermittent) and mitoxantrone, and trended toward improvements versus paclitaxel, docetaxel, and other monotherapy or combination chemotherapy agents. Findings from the random-effects models suggest that palbociclib + letrozole is associated with improved PFS/ TTP relative to all other treatments, although not statistically significant.

The second-line NMA results suggest that palbociclib + fulvestrant is associated with improved PFS/TTP relative to all other chemotherapy treatments. In the fixedeffects model, statistically significant improvements in PFS/TTP were observed in favor of palbociclib + fulvestrant relative to capecitabine (intermittent and continuous), mitoxantrone, and pegylated liposomal doxorubicin, and trended toward improvements versus paclitaxel, docetaxel, and other monotherapy or combination chemotherapy agents. Results from the random-effects models aligned closely with those of the fixed-effects model.

\section{Strengths and limitations}

Palbociclib is a relatively new targeted therapy with the Palbociclib Clinical Trial Development Program still ongoing, and it is currently the only CDK inhibitor approved for use in the US. Since direct head-to-head comparisons have not been made between palbociclib and chemotherapy agents, the current NMA sought to indirectly compare these therapies. To the best of our knowledge, this is the most up-to-date systematic review and NMA to synthesize data for this population of patients with $\mathrm{HR}+$ / HER2 - ABC/MBC. Notably, analyses were stratified by first and second line of therapy rather than considering both populations simultaneously, as was done in the NMA by Generali et al. [7]. Combining first- and second-line therapies likely violates the exchangeability assumption [13], whereas the current stratified approach adheres to best practices for the conduct of NMA [9, 21]. This study also adheres to PRISMA reporting guidelines (Online Appendix F) [22]. Thorough sensitivity analyses were conducted and yielded similar findings for both the first and second lines of therapy, providing evidence for the robustness of study results.

However, there are a few limitations associated with the analyses employed. Firstly, there is heterogeneity in patient and study characteristics, introduced primarily by the fact that the included studies span several decades. The studies included in our analyses were published between 1992 and 2016, so there is likely some heterogeneity in the diagnostic procedures that were used. Stage migration via technology may result in more patients being diagnosed with advanced stages of disease in more recent trials, which may bias survival rates. However, the structure of the evidence networks limited our ability to adjust for these factors. Despite these issues, considerable effort was taken to account for heterogeneity and inconsistency using best practices $[21,22]$ and approaches that are analogous to or exceed those employed by other HTA bodies [23, 24]. Various sensitivity analyses were performed, all of which yielded similar findings to the main analyses. Secondly, although analyses were stratified by line of therapy, some connections had to be forced to maintain a connected network. For example, the study by Bachelot et al. [25] was classified as a second-line study, but it was also forced into first-line networks so that chemotherapy agents of interest, such as docetaxel and paclitaxel, could be included. Three studies were also forced into networks based on patient characteristics: Ackland et al. [17], Dixon et al. [19], and Paridaens et al. [18]. Although the study by Dixon et al. [19] is the oldest study included in our analyses, it was also included by Generali et al. [7] and it appears to be the only appropriate trial available that directly compares an endocrine therapy with a chemotherapy. However, only 20-27\% of patients in this study were estrogen receptor-positive $(\mathrm{ER}+)$, and several key study design characteristics were not reported, including randomization technique, concealment of treatment allocation, or blinding of participants and outcome assessors. Therefore, there is an elevated risk of bias associated with this trial.

Network meta-analyses were also conducted for overall survival; however, these results have been excluded from the current analysis due to immature data in the palbociclib clinical trials.

\section{Conclusions}

Palbociclib + letrozole and palbociclib + fulvestrant demonstrate trends in incremental efficacy compared with chemotherapy agents for the first- and second-line treatment of postmenopausal HR+/HER2- ABC/MBC. Both palbociclib combination therapies consistently showed statistically significant improvements in PFS/TTP versus capecitabine and mitoxantrone, and trended toward improvements versus paclitaxel, docetaxel, and other monotherapy or combination chemotherapy agents. Findings from network meta-analyses were robust to sensitivity analyses, lending credibility to the analyses and conclusions. 
Acknowledgements This study was sponsored by Pfizer, Inc. The authors would like to acknowledge Dagmara Chojecki (contract information specialist for Cornerstone Research Group) for conducting the literature search.

Funding This study was sponsored by Pfizer, Inc.

\section{Compliance with ethical standards}

Conflicts of interest Debanjali Mitra and Shrividya Iyer are employees of Pfizer, Inc. Florence R. Wilson, Abhishek Varu, and Chris Cameron are employees of Cornerstone Research Group, Inc., who were paid contractors to Pfizer in the development of this manuscript. This research was performed under a research contract between Cornerstone Research Group and Pfizer, and was funded by Pfizer.

Open Access This article is distributed under the terms of the Creative Commons Attribution 4.0 International License (http://crea tivecommons.org/licenses/by/4.0/), which permits unrestricted use, distribution, and reproduction in any medium, provided you give appropriate credit to the original author(s) and the source, provide a link to the Creative Commons license, and indicate if changes were made.

\section{References}

1. Cardoso F, Costa A, Norton L, Senkus E, Aapro M, Andre F, Barrios CH, Bergh J, Biganzoli L, Blackwell KL, Cardoso MJ, Cufer T, El Saghir N, Fallowfield L, Fenech D, Francis P, Gelmon K, Giordano SH, Gligorov J, Goldhirsch A, Harbeck N, Houssami N, Hudis C, Kaufman B, Krop I, Kyriakides S, Lin UN, Mayer M, Merjaver SD, Nordstrom EB, Pagani O, Partridge A, Penault-Llorca F, Piccart MJ, Rugo H, Sledge G, Thomssen C, Van't Veer L, Vorobiof D, Vrieling C, West N, Xu B, Winer E (2014) ESO-ESMO 2nd international consensus guidelines for advanced breast cancer (ABC2). Ann Oncol 25(10):1871-1888. doi:10.1093/annonc/mdu385

2. Migliaccio I, Malorni L, Hart CD, Guarducci C, Di Leo A (2015) Endocrine therapy considerations in postmenopausal patients with hormone receptor positive, human epidermal growth factor receptor type 2 negative advanced breast cancers. BMC Med 13(1):1-6. doi:10.1186/s12916-015-0280-0

3. Joy AA, Ghosh M, Fernandes R, Clemons MJ (2015) Systemic treatment approaches in her2-negative advanced breast cancerguidance on the guidelines. Curr Oncol 22(Suppl 1):S29-S42. doi: $10.3747 / \mathrm{co} .22 .2360$

4. Wolters R, Regierer AC, Schwentner L, Geyer V, Possinger K, Kreienberg R, Wischnewsky MB, Wockel A (2012) A comparison of international breast cancer guidelines-do the national guidelines differ in treatment recommendations? Eur J Cancer 48(1):1-11. doi:10.1016/j.ejca.2011.06.020

5. Finn RS, Martin M, Rugo H, Jones S, Im SA, Gelmon K, Harbeck N, Lipatov ON, Walshe JM, Moulder S, Gauthier E, Lu DR, Randolph S, Dieras V, Slamon DJ (2016) Palbociclib and letrozole in advanced breast cancer. N Engl J Med 375(20): 1925-1936

6. Cristofanilli M, Turner NC, Bondarenko I, Ro J, Im SA, Masuda N, Colleoni M, DeMichele A, Loi S, Verma S, Iwata H, Harbeck N, Zhang K, Theall KP, Jiang Y, Bartlett CH, Koehler M, Slamon D (2016) Fulvestrant plus palbociclib versus fulvestrant plus placebo for treatment of hormone-receptor-positive, HER2-negative metastatic breast cancer that progressed on previous endocrine therapy (PALOMA-3): final analysis of the multicentre, double-blind, phase 3 randomised controlled trial. Lancet Oncol. doi:10.1016/S1470-2045(15)00613-0

7. Generali D, Venturini S, Rognoni C, Ciani O, Pusztai L, Loi S, Jerusalem G, Bottini A, Tarricone R (2015) A network metaanalysis of everolimus plus exemestane versus chemotherapy in the first- and second-line treatment of estrogen receptor-positive metastatic breast cancer. Breast Cancer Res Treat 152(1):95-117. doi:10.1007/s10549-015-3453-9

8. Chirila C, Mitra D, Colosia A, Ling C, Odom D, Iyer S, Kaye JA (2017) Comparison of palbociclib in combination with letrozole or fulvestrant with endocrine therapies for advanced/metastatic breast cancer: network meta-analysis. Curr Med Res Opin. doi:10.1080/03007995.2017.1325730

9. Dias S, Sutton AJ, Ades AE, Welton NJ (2013) Evidence synthesis for decision making 2: a generalized linear modeling framework for pairwise and network meta-analysis of randomized controlled trials. Med Decis Making 33(5):607-617. doi:10. 1177/0272989X12458724

10. Dias S, Welton NJ, Sutton AJ, Ades AE (2013) Evidence synthesis for decision making 1: introduction. Med Decis Making 33(5):597-606. doi:10.1177/0272989X13487604

11. Salanti G, Ades AE, Ioannidis JP (2011) Graphical methods and numerical summaries for presenting results from multiple-treatment meta-analysis: an overview and tutorial. J Clin Epidemiol 64(2):163-171. doi:10.1016/j.jclinepi.2010.03.016

12. Rhodes KM, Turner RM, Higgins JP (2015) Predictive distributions were developed for the extent of heterogeneity in metaanalyses of continuous outcome data. J Clin Epidemiol 68(1):52-60. doi:10.1016/j.jclinepi.2014.08.012

13. Cameron C, Fireman B, Hutton B, Clifford T, Coyle D, Wells G, Dormuth CR, Platt R, Toh S (2015) Network meta-analysis incorporating randomized controlled trials and non-randomized comparative cohort studies for assessing the safety and effectiveness of medical treatments: challenges and opportunities. Syst Rev 4:147. doi:10.1186/s13643-015-0133-0

14. Dias S, Sutton AJ, Welton NJ, Ades AE (2013) Evidence synthesis for decision making 3: heterogeneity-subgroups, meta-regression, bias, and bias-adjustment. Med Decis Making 33(5):618-640. doi:10.1177/0272989X13485157

15. Finn RS, Crown JP, Lang I, Boer K, Bondarenko IM, Kulyk SO, Ettl J, Patel R, Pinter T, Schmidt M, Shparyk Y, Thummala AR, Voytko NL, Fowst C, Huang X, Kim ST, Randolph S, Slamon DJ (2015) The cyclin-dependent kinase $4 / 6$ inhibitor palbociclib in combination with letrozole versus letrozole alone as first-line treatment of oestrogen receptor-positive, HER2-negative, advanced breast cancer (PALOMA-1/TRIO-18): a randomised phase 2 study. Lancet Oncol 16(1):25-35. doi:10.1016/S14702045(14)71159-3

16. Dias S, Welton NJ, Sutton AJ, Caldwell DM, Lu G, Ades AE (2013) Evidence synthesis for decision making 4: inconsistency in networks of evidence based on randomized controlled trials. Med Decis Making 33(5):641-656. doi:10.1177/0272989X 12455847

17. Ackland SP, Anton A, Breitbach GP, Colajori E, Tursi JM, Delfino C, Efremidis A, Ezzat A, Fittipaldo A, Kolaric K, Lopez M, Viaro D (2001) Dose-intensive epirubicin-based chemotherapy is superior to an intensive intravenous cyclophosphamide, methotrexate, and fluorouracil regimen in metastatic breast cancer: a randomized multinational study. J Clin Oncol 19(4): 943-953

18. Paridaens R, Biganzoli L, Bruning P, Klijn JG, Gamucci T, Houston S, Coleman R, Schachter J, Van Vreckem A, Sylvester R, Awada A, Wildiers J, Piccart M (2000) Paclitaxel versus doxorubicin as first-line single-agent chemotherapy for metastatic breast cancer: a European Organization for Research and 
Treatment of Cancer Randomized Study with cross-over. J Clin Oncol 18(4):724-733

19. Dixon AR, Jackson L, Chan S, Haybittle J, Blamey RW (1992) A randomised trial of second-line hormone vs single agent chemotherapy in tamoxifen resistant advanced breast cancer. $\mathrm{Br} \mathbf{J}$ Cancer 66(2):402-404

20. Wang J, Xu B, Yuan P, Ma F, Li Q, Zhang P, Cai R, Fan Y, Luo Y, Li Q (2015) Capecitabine combined with docetaxel versus vinorelbine followed by capecitabine maintenance medication for first-line treatment of patients with advanced breast cancer: phase 3 randomized trial. Cancer 121(19):3412-3421. doi:10.1002/ cncr.29492

21. Jansen JP, Trikalinos T, Cappelleri JC, Daw J, Andes S, Eldessouki R, Salanti G (2014) Indirect treatment comparison/network meta-analysis study questionnaire to assess relevance and credibility to inform health care decision making: an ISPOR-AMCPNPC Good Practice Task Force report. Value Health 17(2):157-173. doi:10.1016/j.jval.2014.01.004

22. Hutton B, Salanti G, Caldwell DM, Chaimani A, Schmid CH, Cameron C, Ioannidis JP, Straus S, Thorlund K, Jansen JP, Mulrow C, Catala-Lopez F, Gotzsche PC, Dickersin K, Boutron I,
Altman DG, Moher D (2015) The PRISMA extension statement for reporting of systematic reviews incorporating network metaanalyses of health care interventions: checklist and explanations. Ann Intern Med 162(11):777-784. doi:10.7326/M14-2385

23. McIntosh B, Cameron C, Singh SR, Yu C, Ahuja T, Welton NJ, Dahl M (2011) Second-line therapy in patients with type 2 diabetes inadequately controlled with metformin monotherapy: a systematic review and mixed-treatment comparison meta-analysis. Open Med 5(1):e35-e48

24. Cameron C, Coyle D, Richter T, Kelly S, Gauthier K, Steiner S, Carrier M, Coyle K, Bai A, Moulton K, Clifford T, Wells G (2014) Systematic review and network meta-analysis comparing antithrombotic agents for the prevention of stroke and major bleeding in patients with atrial fibrillation. BMJ Open 4(6):e004301. doi:10.1136/bmjopen-2013-004301

25. Bachelot T, Bajard A, Ray-Coquard I, Provencal J, Coeffic D, Agostini C, Boisseau M, Kaphan R, Dramais D, Oprea C, FerriDessens RM, Guastalla JP, Perol D (2011) Final results of ERASME-4: a randomized trial of first-line docetaxel plus either capecitabine or epirubicin for metastatic breast cancer. Oncology 80(3-4):262-268. doi:10.1159/000329066 